\title{
Direct synthesis of few- and multi-layer graphene films on dielectric substrates by "etching-precipitation" method
}

\author{
Masaki Kosaka $^{a, b}$, Soichiro Takano ${ }^{a}$, Kei Hasegawa ${ }^{b}$, Suguru Noda $^{b}{ }^{*}$ \\ ${ }^{a}$ Department of Chemical System Engineering, School of Engineering, The University of \\ Tokyo, 7-3-1 Hongo, Bunkyo-ku, Tokyo 113-8656, Japan \\ ${ }^{b}$ Department of Applied Chemistry, School of Advanced Science and Engineering, Waseda \\ University, 3-4-1 Okubo, Shinjuku-ku, Tokyo 169-8555, Japan
}

\begin{abstract}
A novel "etching-precipitation" method is proposed and developed for the direct synthesis of graphene on dielectric substrates. In this method, graphene precipitates from the $\mathrm{Fe}-\mathrm{C}$ solid solution film during selective etching of $\mathrm{Fe}$ using $\mathrm{Cl}_{2}$ gas. Few- and multi-layer graphene is fabricated directly on quartz glass and $\mathrm{SiO}_{2} / \mathrm{Si}$ substrates without $\mathrm{Fe}$ residue at a growth temperature of $500-650{ }^{\circ} \mathrm{C}$, which is a significantly lower temperature than used in the conventional chemical vapor deposition method. The 6- to 7-layer graphene synthesized at $650{ }^{\circ} \mathrm{C}$ shows a volume resistivity of $80-140 \mu \Omega \mathrm{cm}$. The average number of layers can be easily controlled in a linear fashion with the initial carbon feed, which is proportional to the thickness of the starting Fe-C films. Line-patterned multi-layer graphene is also fabricated by simply pre-patterning the starting Fe-C film although its structure is somewhat different from typical graphene ribbons. "Etching-precipitation" will be a practical route to synthesize graphene with micro-patterns directly onto device substrates of arbitrary sizes.
\end{abstract}

\footnotetext{
* Corresponding author: Tel/Fax: +81-3-5286-2769.

E-mail address: noda@waseda.jp (S. Noda).
} 


\section{Introduction}

Graphene [1], which is a monolayer or few layers of $\mathrm{sp}^{2}$ bonded carbon atoms, has been attracting much attention since Novoselov et al. revealed its unique properties in 2004 [2]. Graphene's tunable energy band structure, high carrier mobility, mechanical/chemical stability, and acceptable optical transparency can enable various applications such as transparent electrodes, transistors, and wirings in large-scale integrated circuits (LSIs) by adjusting the layer number [3-5]. Therefore, fabrication methods that provide good control over the structure and layer number of graphene have been extensively explored. Currently, chemical vapor deposition (CVD) on catalyst metals [6-20] is considered the most promising method for the synthesis of graphene because of its high scalability. Graphene films 30 inches in size were produced for transparent electrodes by CVD on $\mathrm{Cu}$ foils, and transparent electrodes having a sheet resistance of $30 \Omega / \mathrm{sq}$. and an optical transmittance of $90 \%$ (excluding the absorption by the base film) were fabricated by transferring graphene four times to polyethylene terephthalate films [7]. For many applications, it is necessary to have graphene on dielectric substrates. The transfer of graphene to such substrates has been realized by complete etching of the catalyst metals by liquid etchants (e.g., $\mathrm{FeCl}_{3}$ as) [7-18] and, more recently, by the electrochemical delamination or electrolysis-based bubbling transfer method [21,22]. However, wet processes can easily cause impurities and damage to graphene, resulting in an increase in resistivity or degradation of mobility. Furthermore, it is very difficult to fabricate graphene with a micro-pattern on a patterned underlayer, such as is necessary for creating wiring in LSIs. To solve the transfer process difficulties, some groups have recently reported the synthesis of graphene between the catalyst metals and the dielectric substrates [23-27], and the synthesis of graphene by CVD without a metal catalyst [28,29]. However, the synthesis of graphene possessing an acceptable level of quality and uniformity directly on dielectric substrates is still a challenge, and a breakthrough toward obtaining good 
controllability over the layer number and micro-patterning is in high demand.

In this study, we propose an "etching-precipitation" method that enables the direct synthesis of metal-free graphene on dielectric substrates. Fig. 1 is a schematic comparing the etching-precipitation method with the ordinary CVD method. Two types of metal catalysts are used for CVD. In the case of carbon-insoluble metals (e.g., $\mathrm{Cu}$ ) [6-12], carbon feedstock (e.g., $\mathrm{CH}_{4}$ ) decomposes on the catalyst surface and forms graphene through a surface reaction mechanism [11] (Fig. 1a). In the case of carbon-soluble metals (e.g., Ni, Co, and Fe) [11-18], the carbon feedstock decomposes on and dissolves into the catalyst, and the carbon precipitating from the catalyst upon cooling [11] predominantly contributes to the graphene growth with some contribution from the surface reaction mechanism [30] (Fig. 1b). In case of CVD at low temperatures, these metals have lower solubility of carbon and yield graphene also via the surface reaction mechanism, as previously reported for the $\mathrm{Ni}$ - $\mathrm{Au}$ alloy catalyst at $450{ }^{\circ} \mathrm{C}$ [31]. Uniform monolayer graphene was recently realized through the surface reaction mechanism on the $\mathrm{Ni}-\mathrm{Au}$ ally catalyst by $\mathrm{CVD}$ at $600{ }^{\circ} \mathrm{C}$ via the careful control over the surface reaction and bulk diffusion kinetics [32]. For the case of CVD at high temperatures, the carbon supersaturation that occurs because of the reduced solubility of carbon in catalyst metals during cooling drives the carbon precipitation from the metal-carbon solid solution [11]. More precisely, the driving force for the precipitation of carbon is the lower chemical potential of carbon in graphene/graphite than that in the supersaturated metal-carbon solid solution. This gives birth to the new idea of driving the precipitation of carbon not by decreasing the solubility of carbon, but instead by increasing the carbon concentration in the solid solution. We propose the etching-precipitation method for graphene synthesis in which the solute carbon is supersaturated by removing the solvent metal from the "hot metal-carbon solid solution" through dry-etching (Fig. 1c). We can choose the metal from the carbon soluble metals such as $\mathrm{Fe}, \mathrm{Co}$, and $\mathrm{Ni}$, and Fe having a high carbon solubility (up to 25 at\% in 
a form of $\mathrm{Fe}_{3} \mathrm{C}$ carbide) is selected in this work to achieve rather thick graphene for possible application to wiring in LSIs. The Fe-C mixture film is deposited on either quartz glass or an $\mathrm{SiO}_{2} / \mathrm{Si}$ substrate and heated to $400-700{ }^{\circ} \mathrm{C}$, whereupon the $\mathrm{Fe}$ is gradually etched away by $\mathrm{Cl}_{2}$ gas to yield graphene directly on the $\mathrm{SiO}_{2} / \mathrm{Si}$ without metal residue. Few-layer graphene and multi-layer graphene, which are defined as graphene having 2-5 and 2-10 layers, respectively [1], can be obtained with a moderate process temperature of $500-650{ }^{\circ} \mathrm{C}$ because the graphene growth proceeds at a constant temperature, thereby keeping the carbon mobile. Furthermore, control over the number of graphene layers as well as patterned growth, which are important base technologies for wiring in LSIs, are realized by tailoring the structure of the initial $\mathrm{Fe}-\mathrm{C}$ mixture films.

(a)

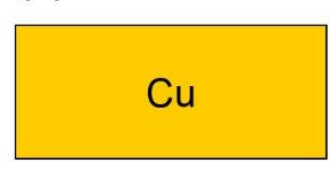

(b)

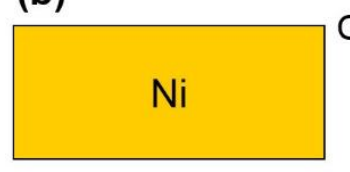

(c)

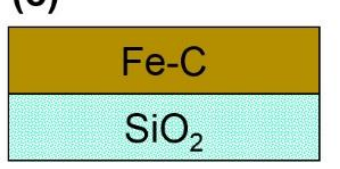

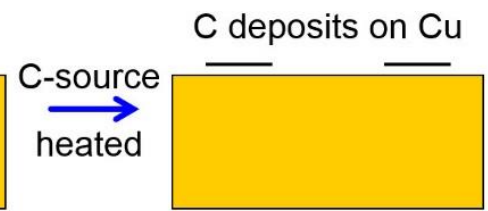
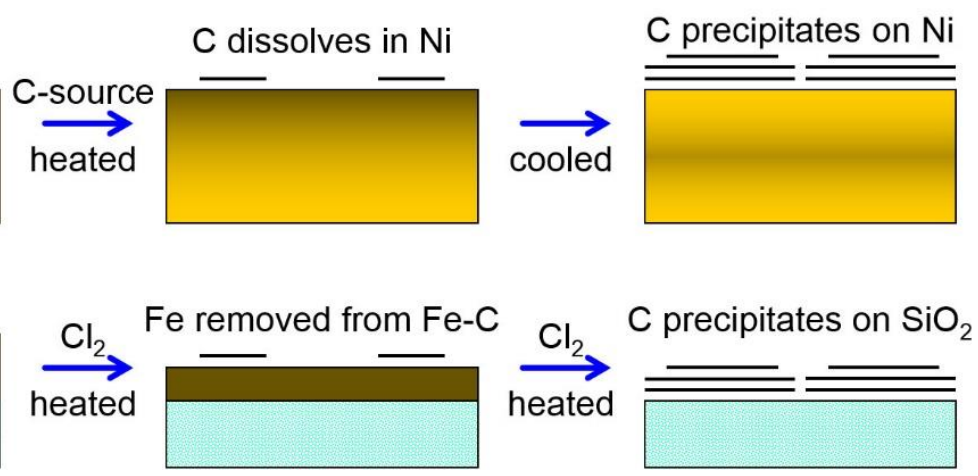

Fig. 1 - Schematic comparing the CVD and "etching-precipitation" processes. (a) In a CVD process using a carbon-insoluble metal catalyst such as $\mathrm{Cu}$, the graphene growth proceeds through the surface reaction [11]. (b) In a CVD process using a carbon-soluble metal catalyst such as $\mathrm{Ni}$, the graphene growth proceeds through carbon supersaturation and precipitation from the Ni-C solid solution upon cooling [11], as well as through the surface reaction [30-32]. (c) In the etching-precipitation of the metal-C mixed film (e.g., metal=Fe), the graphene growth proceeds through carbon supersaturation and precipitation by removing solid-solvent $\mathrm{Fe}$, resulting in Fe-free 
graphene directly on the substrates.

\section{Experimental}

Quartz glass substrates or Si substrates with a 90-nm-thick thermal oxide layer were used as substrates. The substrates were pretreated by dipping them into either a $1 \mathrm{wt} \% \mathrm{HF}$ solution or a mixed solution of $\mathrm{H}_{2} \mathrm{SO}_{4}(95 \mathrm{wt} \%)$ and $\mathrm{H}_{2} \mathrm{O}_{2}(30 \mathrm{wt} \%)$ with a volume ratio of 3:1, followed by washing with deionized water. On the substrates, an Fe-C mixture film (20$200 \mathrm{~nm}$ in thickness) was deposited by DC magnetron sputtering under 3-10 mTorr Ar using a pure Fe target $(0.5 \mathrm{~mm}$ thick, 2 inch diameter $)$ on which carbon chips $\left(2 \times 12 \mathrm{~mm}^{2}\right.$, eight pieces) were attached using a carbon paste (see Supplementary data, Fig. S1). We adjusted the $\mathrm{C}$ content to make $\mathrm{C}$ concentration not so high in the $\mathrm{Fe}-\mathrm{C}$ mixture (otherwise graphene forms upon annealing without etching), by changing the area of the carbon chip on the Fe target. In a reference experiment, a Fe-C bilayer film was prepared by sequentially depositing amorphous carbon (5-113 nm in thickness) and Fe (1.6-393 nm in thickness) by DC magnetron sputtering under 10 mTorr Ar.

The samples were set in a quartz glass tubular reactor (34-mm inner diameter and 300-mm heating zone length), heated to and annealed for 10-30 min at a target temperature of $400-700{ }^{\circ} \mathrm{C}$ for $\mathrm{Fe}-\mathrm{C}$ mixture films and $700-800{ }^{\circ} \mathrm{C}$ for $\mathrm{Fe}-\mathrm{C}$ bilayers while being exposed to a $25 \mathrm{vol} \% \mathrm{H}_{2} /$ Ar balance mixture at a flow rate of 400 standard cubic centimeter ( $\mathrm{sccm}$ ) and pressure of 100 Torr, thereby making a solid solution. A subsequent dry-etching of the Fe was carried out for $0-90 \mathrm{~min}$ by switching the gas to a $0.01-1$ vol\% (0.05-0.5 vol\% typically) $\mathrm{Cl}_{2} / \mathrm{Ar}$ balance at a total pressure of 5 Torr and the same temperature used for annealing.

The resulting graphene samples were analyzed by micro-Raman scattering spectroscopy (Horiba HR-800, Kyoto, Japan), scanning electron microscopy (SEM; Hitachi S-4800, Tokyo, Japan) with energy dispersive X-ray spectroscopy (EDS; AMETEK EDAX 
Genesis, Berwyn, PA, USA), and atomic force microscope (AFM, Shimadzu SPM-9600, Kyoto, Japan). Sheet resistances of the samples were evaluated by the four probe method. Optical transmittance was evaluated by an optical scanner (Canon Canoscan 9000F, Tokyo, Japan) in the linear scale.

\section{Results and discussion}

\subsection{Direct synthesis of few-/multi-layer graphene on dielectric substrates}

Fig. 2 shows a typical graphene film on quartz glass synthesized by the etching-precipitation method. The 100-nm-thick Fe-C mixture film with an atomic composition of Fe:C:O = 82:10:8, as measured by SEM-EDS, is used. The photograph (Fig. 2a) shows the uniform gray color over the substrate, which suggests a uniform formation of few-/multi-layer graphene. The optical transmittance of the film is $86 \%$ (excluding the absorption by the base quartz glass), indicating an average layer number of 6-7. The Raman spectrum (Fig. 2b) shows a G-band peak and a relatively small D-band peak with a G-to-D intensity ratio $\left(I_{\mathrm{G}} / I_{\mathrm{D}}\right)$ of 20 . The plan-view SEM image (Fig. 2c) has some contrast variation, which suggests a distribution of the layer number, and the presence of small holes suggests the presence of voids in the graphene film. From this image, the domain size and the graphene coverage are estimated to be $4-5 \mu \mathrm{m}$ and over $99 \%$, respectively. AFM images are taken for the graphene samples transferred from the quartz glass substrate (rough surface) to a $\mathrm{SiO}_{2} / \mathrm{Si}$ substrate (flatter surface) by partial etching of the quartz glass using a dilute hydrofluoric acid. From the AFM image taken near the voids (Fig. 2d), the height difference between the graphene and the void surfaces (Positions A and B) is $3.4 \mathrm{~nm}$ on average, which is somewhat larger than the thickness of $\sim 2 \mathrm{~nm}$ expected from the layer number of $6-7$. In addition to the layer number distribution, some wrinkles are observed, which may cause the increase in the graphene film thickness. This film has a sheet resistance of $340-460 \Omega / \mathrm{sq}$., and the volume 
resistivity is estimated to be in a range from $\sim 80 \mu \Omega \mathrm{cm}$, using the thickness of $2 \mathrm{~nm}$ obtained from the optical transmittance, to $\sim 140 \mu \Omega \mathrm{cm}$, using the thickness of $3.4 \mathrm{~nm}$ from the AFM measurement. These results show that a multi-layer graphene film is synthesized directly on the dielectric substrate via our etching-precipitation method.
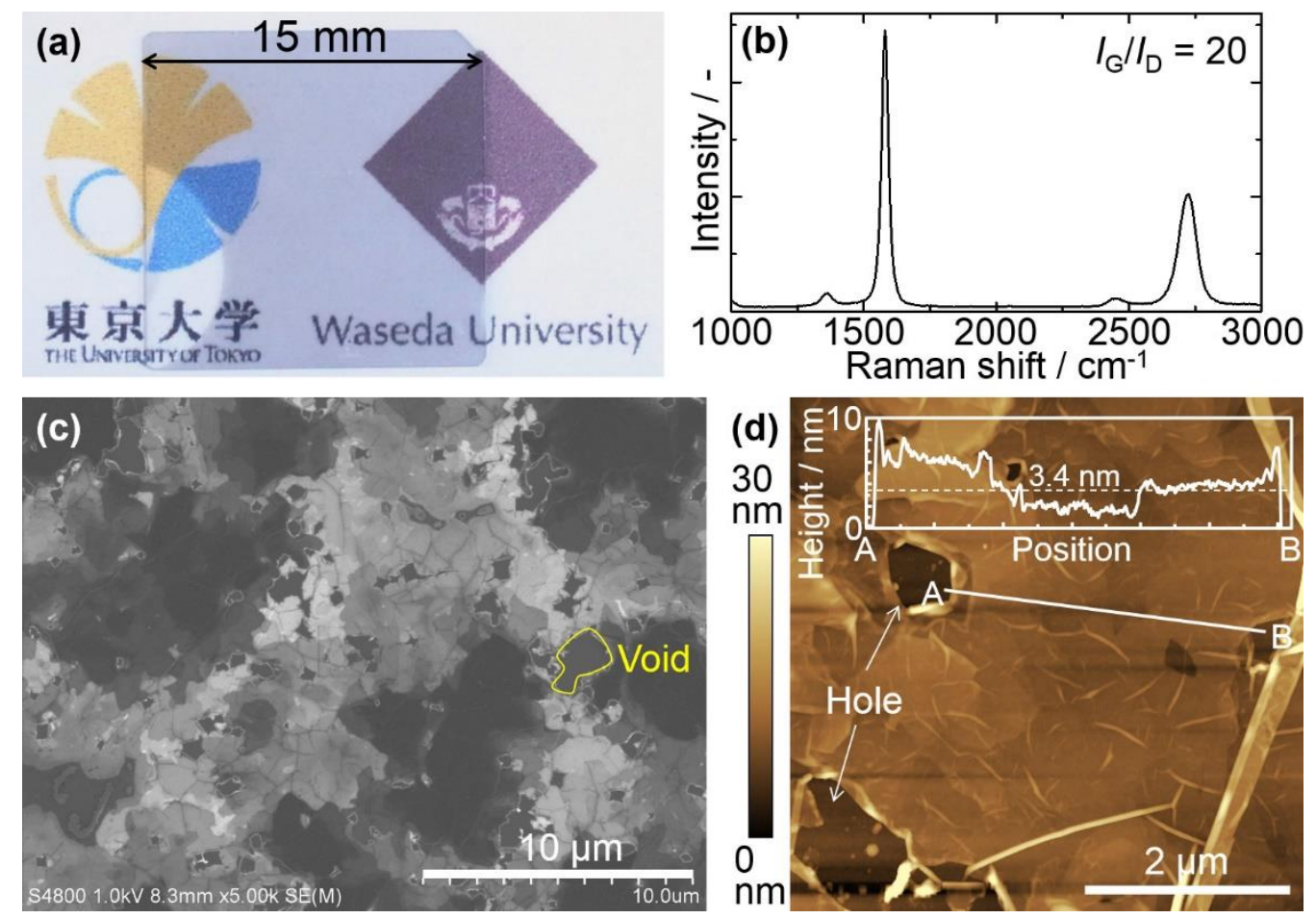

Fig. 2 - The (a) photograph, (b) Raman spectrum, (c) SEM image, and (d) AFM image of a typical multi-layer graphene sample synthesized by the etching-precipitation method. The 100-nm-thick Fe-C mixture film on a quartz glass substrate is etched under 0.05 vol\% $\mathrm{Cl}_{2}$ at 5 Torr and $650{ }^{\circ} \mathrm{C}$ for $60 \mathrm{~min}$. The graphene is $86 \%$ transparent, corresponding to six to seven layers, $3.4 \mathrm{~nm}$ in thickness, and a sheet resistance of 340$460 \Omega / \mathrm{sq}$., which corresponds to $80-140 \mu \Omega \mathrm{cm}$ volume resistivity (see the main text). The Raman spectrum is taken at $488 \mathrm{~nm}$ for excitation. The AFM image is obtained from graphene transferred onto a $\mathrm{SiO}_{2} / \mathrm{Si}$ substrate with a flat surface.

3.2. Effect of the etching temperature: competition between graphene domain growth and dewetting of Fe-C films 
Next, we discuss the effect of etching temperature upon the structure and quality of graphene. In the case of conventional CVD, a high temperature is required to grow high-quality graphene, with typical growth temperatures of $1000{ }^{\circ} \mathrm{C}$ or above on $\mathrm{Cu}[5-11]$ and $900-1000{ }^{\circ} \mathrm{C}$ on $\mathrm{Ni}[10,12-14]$. With direct growth of graphene on devices, however, the upper limit of the process temperature is often much lower. In the case of LSIs, it is normally as low as $400{ }^{\circ} \mathrm{C}$. In our etching-precipitation method, the graphene growth can be driven at a fixed temperature without cooling, so graphene can be grown even at low process temperatures.

Fig. 3 shows the few-/multi-layer graphene samples grown at $400-700{ }^{\circ} \mathrm{C}$. The plan-view SEM images (Fig. 3a-g) show drastic changes in the films with growth temperature. A very fine structure is seen at lower temperatures $\left(400-500{ }^{\circ} \mathrm{C}\right)$, a domain structure with larger domain sizes at higher temperatures $\left(550-650{ }^{\circ} \mathrm{C}\right)$ and, finally, a discontinuous film at the highest temperature $\left(700^{\circ} \mathrm{C}\right)$. The Raman spectra (Fig. 3h) also changes drastically with growth temperature, with unseparated, broad D-band and G-band peaks suggesting amorphous carbon at low temperatures $\left(400-450{ }^{\circ} \mathrm{C}\right)$, and a sharp G-band peak with a weak D-band peak suggesting graphitized carbon at high temperatures $\left(500-700{ }^{\circ} \mathrm{C}\right)$. The coverage values and the $I_{\mathrm{G}} / I_{\mathrm{D}}$ values are summarized in Fig. 3i. At growth temperatures $400-450{ }^{\circ} \mathrm{C}$, the $I_{\mathrm{G}} / I_{\mathrm{D}}$ is around unity, revealing the formation of amorphous carbon. The $I_{\mathrm{G}} / I_{\mathrm{D}}$ increases to $\sim 5$ and above at $500{ }^{\circ} \mathrm{C}$ and above, revealing the formation of few-/multi-layer graphene. The surface coverage of graphene is near $100 \%$ for growth temperatures of $500-650{ }^{\circ} \mathrm{C}$, revealing the formation of continuous graphene films; but a further increase of the growth temperature to $700{ }^{\circ} \mathrm{C}$ results in a drastic decrease of the coverage to $<60 \%$, demonstrating the discontinuous graphene formation due to the dewetting of the initial Fe-C film. The discontinuous film grown at $700{ }^{\circ} \mathrm{C}$ has a smaller $I_{\mathrm{G}} / I_{\mathrm{D}}$ ratio than the continuous film grown 
at $650{ }^{\circ} \mathrm{C}$ because of the formation of voids.
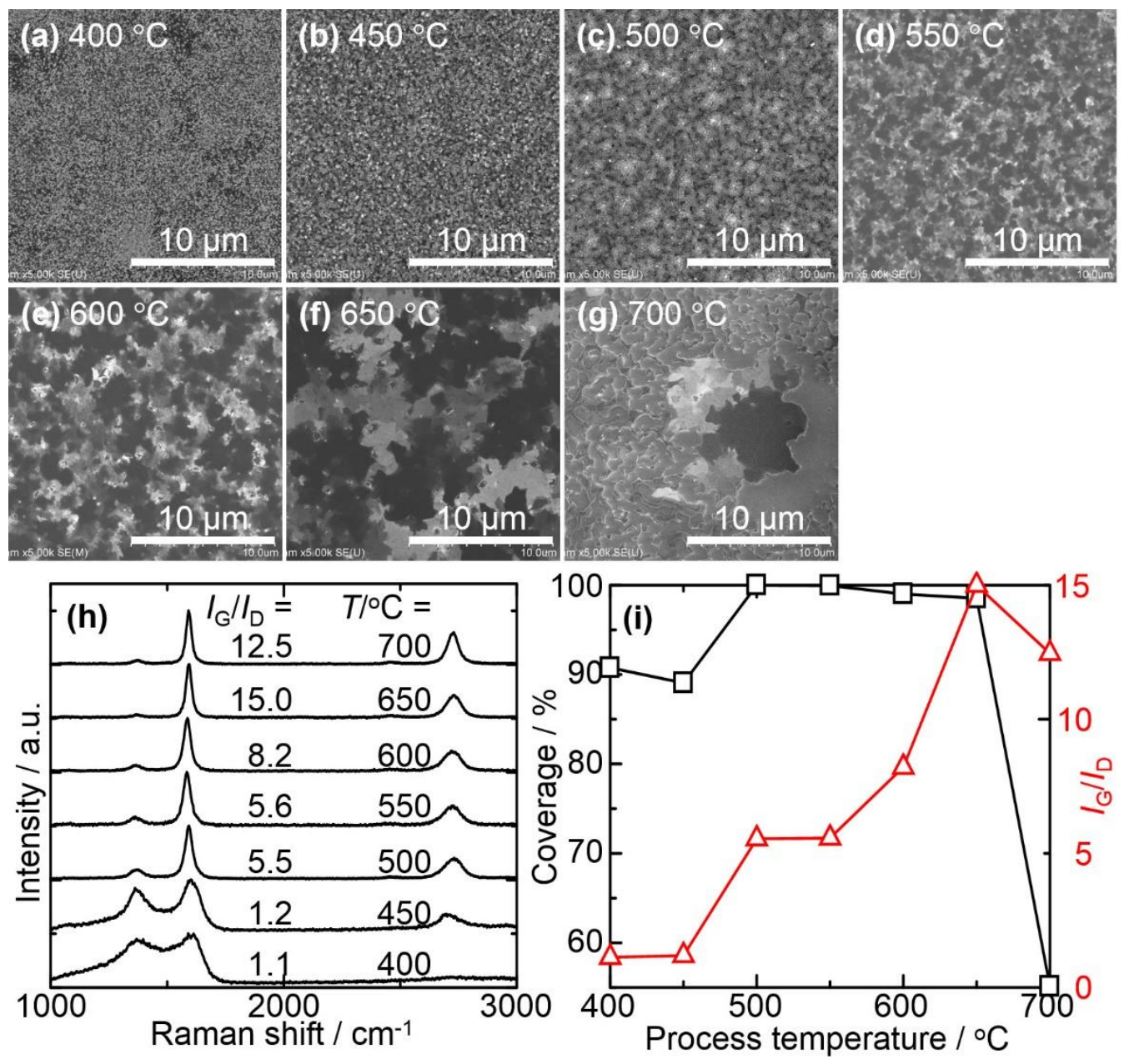

Fig. 3 - Few-/multi-layer graphene synthesized on $\mathrm{SiO}_{2} / \mathrm{Si}$ from a 100-nm-thick Fe-C film by etching-precipitation using 0.1 vol\% $\mathrm{Cl}_{2}$ at 5 Torr for $15 \mathrm{~min}$ at various temperatures. (a-g) Plan-view SEM images. (h) Raman spectra using an excitation wavelength of $488 \mathrm{~nm}$. (i) The surface coverage and the $I_{G} / I_{D}$ ratios of the graphene films synthesized at $400-700{ }^{\circ} \mathrm{C}$.

These structural changes as a function of temperature can be understood by the diffusion of carbon and the dewetting of Fe-C films. The diffusion coefficient, and thus the diffusion length of carbon atoms in $\mathrm{Fe}$, is strongly dependent upon growth temperature and about 100 times larger at $700{ }^{\circ} \mathrm{C}$ than at $400{ }^{\circ} \mathrm{C}$ [33]. Therefore, the graphene domains grow bigger with fewer defects at higher temperatures. The characteristic time for dewetting of 
$\mathrm{Fe}-\mathrm{C}$ films is also strongly dependent upon the growth temperature, and is less than $15 \mathrm{~min}$ at $700{ }^{\circ} \mathrm{C}$ for the conditions given in Fig. 3. This short characteristic time at $700{ }^{\circ} \mathrm{C}$ produces a film with many voids and edges, resulting in the reduced $I_{\mathrm{G}} / I_{\mathrm{D}}$ ratio. The trade-off relationship at higher temperatures between the improved domain size and crystallinity and the degraded continuity of the graphene is evident. The best balance is achieved with a growth temperature of $600-650{ }^{\circ} \mathrm{C}$ for a 15 -min etching time using $5 \mathrm{mTorr} \mathrm{Cl}_{2}$, though the optimum temperature should be lower for a longer etching time using $\mathrm{Cl}_{2}$ at lower partial pressures. We also applied the etching-precipitation method using a sputtered Fe/amorphous $\mathrm{C}_{\mathrm{SiO}}$ multilayer (Fig. S2). For this multilayer, the diffusion length for mixing between the Fe and $\mathrm{C}$ layers is much larger than that for the mixed Fe-C films, creating the need for a higher process temperature and resulting in the dewetting of the Fe-C layer and graphitic films with many voids present.

\subsection{Control of the graphene layer number by the initial carbon feed}

A method to simply control the number of graphene layers is essential for many graphene applications. Fig. 4 demonstrates our results for a simple control method of the graphene layer number by adjusting the initial carbon feed via varying the $\mathrm{Fe}-\mathrm{C}$ film thickness. In Fig. 4a, we can see that the graphene films on quartz glass substrates are uniform in color, and are darker for thicker initial Fe-C films. The average layer number of graphene, as estimated from the optical transmittance, increases linearly with the film thickness and, therefore, the amount of carbon feed, indicating the simplicity of our method (Fig. 4b). The graphene film grown from the 100-nm-thick Fe-C film has an optical transmittance of $86 \%$, corresponding to six to seven layers and a thickness of $\sim 2 \mathrm{~nm}$. Whereas the initial carbon feed in the 100-nm-thick Fe-C film corresponds to a 10-nm-thick carbon layer. SEM-EDS analysis shows that the $\mathrm{Fe}-\mathrm{C}$ film contains oxygen at $\sim 8$ at $\%$ owing to the background pressure in the sputter deposition system and/or its exposure to the air. It is probable that 8 at $\% \mathrm{C}$ is 
consumed in reducing iron oxide and yielding $\mathrm{CO}$, and the remaining 2 at $\% \mathrm{C}$ is converted to graphene. The number of graphene layers estimated by considering this effect is shown as a solid blue line in Fig. 4b, which matches well with the experimental results (blue open squares). Therefore, if the oxygen content in the Fe-C film is constant, the average layer number can be linearly controlled by the initial carbon feed. Plan-view SEM images are shown in Fig. 4c for the few-/multi-layer graphene films grown from 20-, 35-, 100-, and 200-nm-thick $\mathrm{Fe}-\mathrm{C}$ films on $\mathrm{SiO}_{2} / \mathrm{Si}$ substrates. The few-/multi-layer graphene exhibits a continuous structure when grown from Fe-C films $100 \mathrm{~nm}$ or thicker, whereas it displays a discontinuous structure with voids when grown from Fe-C films $35 \mathrm{~nm}$ or thinner. Both the layer number (Fig. 4b) and the surface coverage (Fig. 4d) of the graphene increases with the initial film thickness, and it remains a challenge to obtain a continuous graphene film of very few layers via this etching-precipitation method. 

(a) Graphene on $40 \mathrm{~nm}$ quartz glass from $20 \mathrm{~nm} \mathrm{Fe}-\mathrm{C}$

(b)

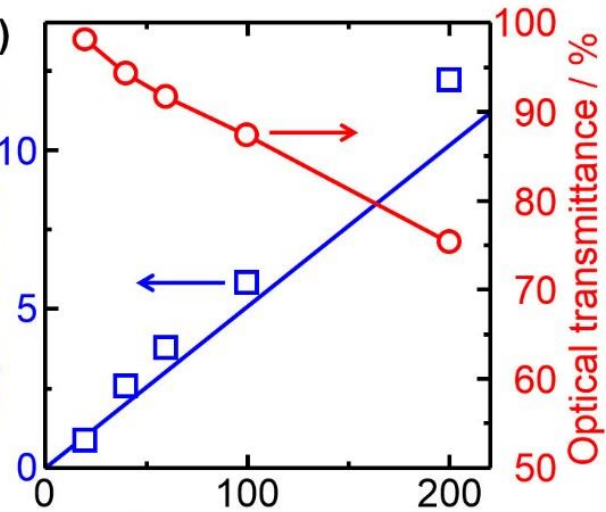

Fe-C film thickness / $\mathrm{nm}$

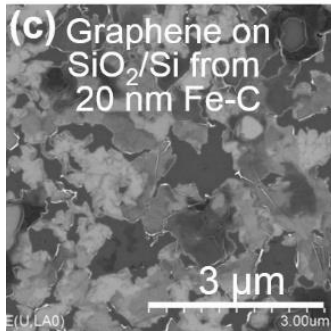

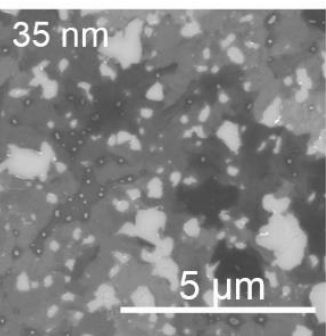

(d)

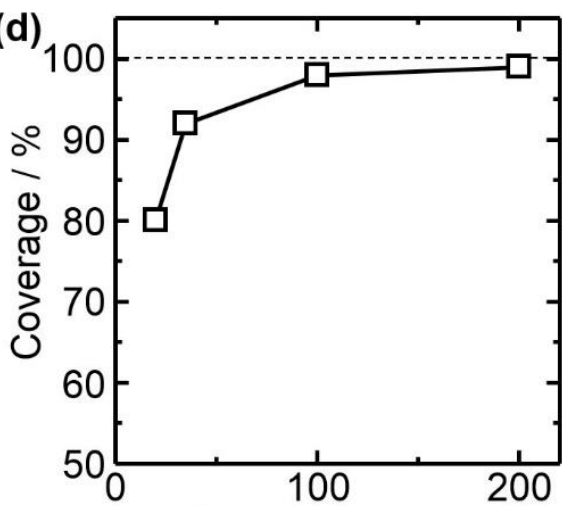

Fe-C film thickness / $\mathrm{nm}$

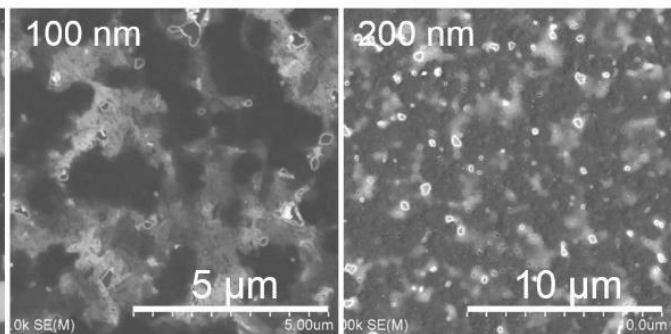

Fig. 4 - (a) Optically scanned images of few-/multi-layer graphene grown from 20 to 200-nm-thick Fe-C at $600{ }^{\circ} \mathrm{C}$ using 0.1 vol\% $\mathrm{Cl}_{2}$ at 5 Torr on quartz glass substrates of $15 \times 15 \mathrm{~mm}^{2}$. Etching was performed for $15 \mathrm{~min}$ for all samples except for 200 -nm-thick Fe-C (90 min) to ensure the complete removal of Fe. (b) Optical transmittance (blue open squares) and layer number (red open circles) of the graphene on quartz glass substrates shown in (a). The blue straight line shows the estimated layer number when considering the mass balance in $\mathrm{C}$ for the reduction of iron oxide and graphene formation (see the main text). (c) Plan-view SEM images of the few-/multi-layer graphene films grown from 20 - to 200 -nm-thick $\mathrm{Fe}-\mathrm{C}$ at $600{ }^{\circ} \mathrm{C}$ on $\mathrm{SiO}_{2} / \mathrm{Si}$ substrates. (d) Surface coverage of the graphene films on $\mathrm{SiO}_{2} / \mathrm{Si}$ substrates shown in (c).

To better understand the dewetting process of the Fe-C films during etching, etching is performed for a short time (2 min) and the surface is characterized by SEM-EDS (Fig. 5). 
The Fe-C film exhibits a discontinuous surface with many holes (Fig. 5a). Each hole shows weak EDS signals for both $\mathrm{C}$ and Fe (Fig. 5b,c) and the SEM images exhibit bright regions at the center surrounded by the dark region (Fig. 5a). The bright regions are charged up and prove to be the voids (i.e., a bare $\mathrm{SiO}_{2}$ surface), and the dark region is the graphene on $\mathrm{SiO}_{2}$ without Fe. These void structures can be explained using the schematic model shown in Fig. 5d. The schematic demonstrates that during the etching-precipitation process, surface tension in the $\mathrm{Fe}-\mathrm{C}$ film drives the film to dewet with time and with the decreasing film thickness. If holes form in the $\mathrm{Fe}-\mathrm{C}$ film at a very early stage prior to the graphene precipitation, a bare $\mathrm{SiO}_{2}$ surface is exposed and remains as voids. As the etching proceeds, the $\mathrm{C}$ concentration in the Fe-C film increases until the $\mathrm{C}$ becomes supersaturated and graphene starts to grow. Near the voids, the graphene sits directly on the $\mathrm{SiO}_{2}$ surface, resulting in the dark region surrounding the voids. For the remaining areas, the graphene sits on the discontinuous Fe-C film and the layer number increases as the etching proceeds. The etching proceeds for Fe even under the graphene layer, possibly via diffusion of $\mathrm{Cl}$ - and/or Fe-species through the graphene at its grain boundaries and/or voids. Finally, the $\mathrm{Fe}-\mathrm{C}$ film is completely etched away, resulting in the Fe-free fw-/multi-layer graphene with some distribution in the layer number (Figs. 2 and 3). The surface coverage of graphene is less for thin $\mathrm{Fe}-\mathrm{C}$ films, which begin to dewet quickly before the graphene starts to precipitate.

We also observed the $\mathrm{Fe}-\mathrm{C}$ surface after annealing without and with etching by Raman spectroscopy (Fig. S3). After annealing and cooling down, a very weak G-band peak (1/30 of the one after 10 min etching) appeared, showing some graphene forming at a very small amount on the Fe-C surface. As the etching proceeds, the D, G, and G'-band peaks got dramatically enhanced, showing that the graphene mainly formed upon etching by $\mathrm{Cl}_{2}$ gas. Differently from the etching-precipitation that yields few-/multi-layer graphene films directly on $\mathrm{SiO}_{2}$ surfaces, the wet-etching of the annealed $\mathrm{Fe}-\mathrm{C}$ film caused the graphene (at a small 
amount) to be lifted-off and to disappear (not to float as a continuous film).
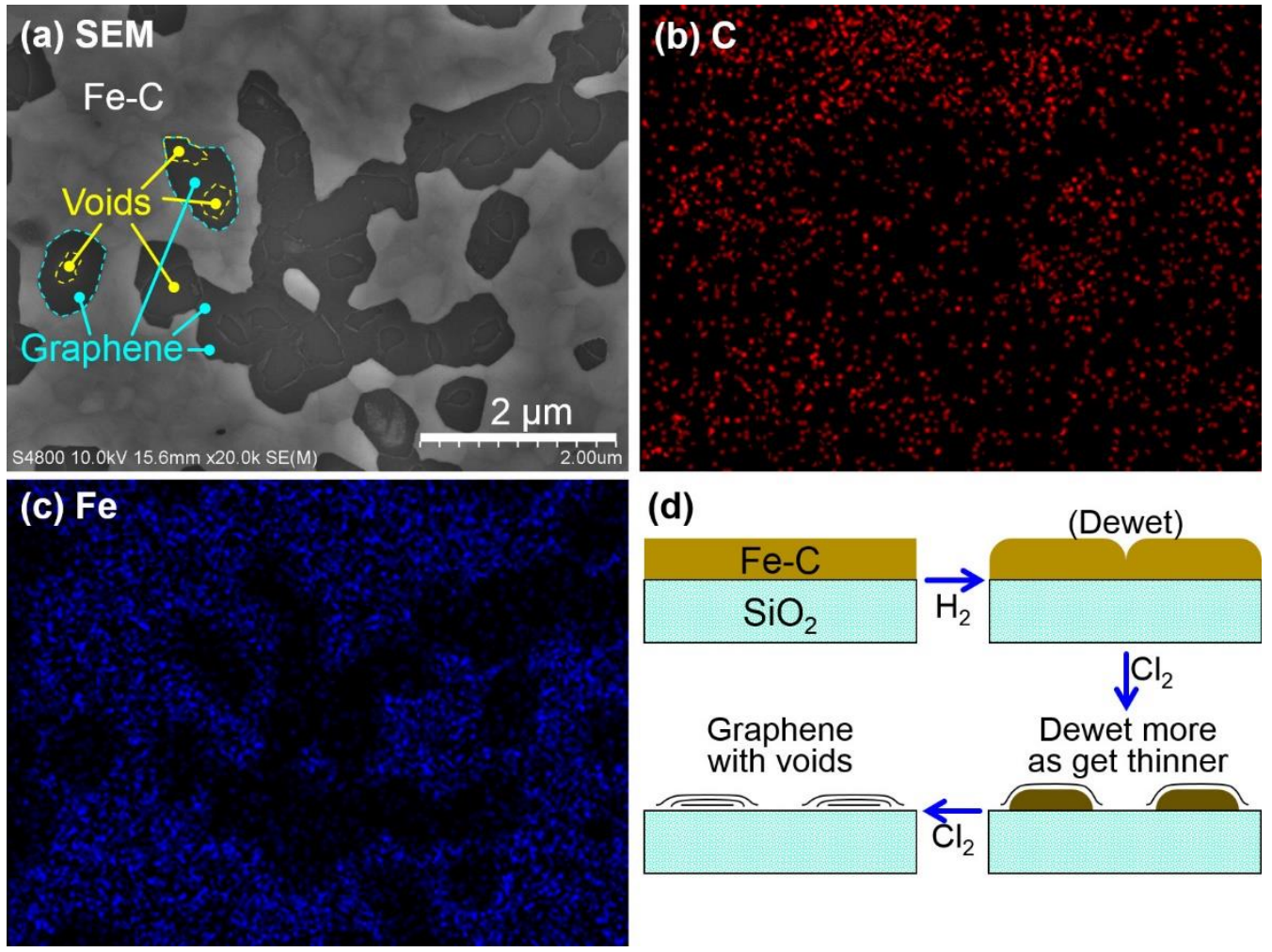

Fig. 5 - (a) SEM images of the 100-nm-thick Fe-C film partially etched by 0.1 vol\% $\mathrm{Cl}_{2}$ for a short time of 2 min at $600{ }^{\circ} \mathrm{C}$, and EDS mapping images of (b) $\mathrm{C}$ and (c) Fe on the surface. Island-like structures of the Fe-C mixture, voids (surrounded by yellow broken curves), and graphene surrounding the voids (the regions between yellow and blue broken curves) are observed. (d) A schematic for the formation mechanism of few-/multi-layer graphene and voids.

The key to fabricating uniform graphene without voids is to suppress the dewetting of the Fe-C films. One approach to prevent the dewetting is to stabilize the interface between the $\mathrm{Fe}-\mathrm{C}$ film and the $\mathrm{SiO}_{2}$ substrate. To stabilize the interface, we use a $\mathrm{Cu}$ as a sacrificial underlayer for the Fe-C film (Fig. 6a). As the etching of the Fe-C film proceeds, the concentration of $\mathrm{C}$ increases in the $\mathrm{Fe}-\mathrm{C}$ film while $\mathrm{C}$ cannot diffuse into $\mathrm{Cu}$ due to the limited $\mathrm{C}$ solubility in it. After the complete etching of the Fe-C film, graphene sits on the $\mathrm{Cu}$ 
underlayer. $\mathrm{Cu}$ is also etched away by $\mathrm{Cl}_{2}$ to yield $\mathrm{Cu}_{n} \mathrm{Cl}_{n}$ gases ( $n=1,3$ as the main species) and finally the graphene sits directly on $\mathrm{SiO}_{2}$. Fig. $6 \mathrm{~b}-\mathrm{e}$ shows the few-/multi-layer graphene synthesized from the 100-nm-thick Fe-C film on 100-nm-thick $\mathrm{Cu}$ sacrificial underlayer on a $\mathrm{SiO}_{2}$ substrate via etching-precipitation at $600^{\circ} \mathrm{C}$. The number of voids drastically decreases with the sacrificial underlayer (Fig. 6b), showing that the suppression of the dewetting of the $\mathrm{Fe}-\mathrm{C}$ film is indeed the key for growing uniform graphene. However, the etching of $\mathrm{Cu}$ is thermodynamically more favorable than Fe, resulting in some irregularly etched regions (Fig. 6d,e). To obtain graphene without voids, it is important to find an underlayer material that improves the interfacial interaction between the $\mathrm{Fe}-\mathrm{C}$ films and $\mathrm{SiO}_{2}$ and is thermodynamically less favorable for etching by $\mathrm{Cl}_{2}$ than $\mathrm{Fe}$. 

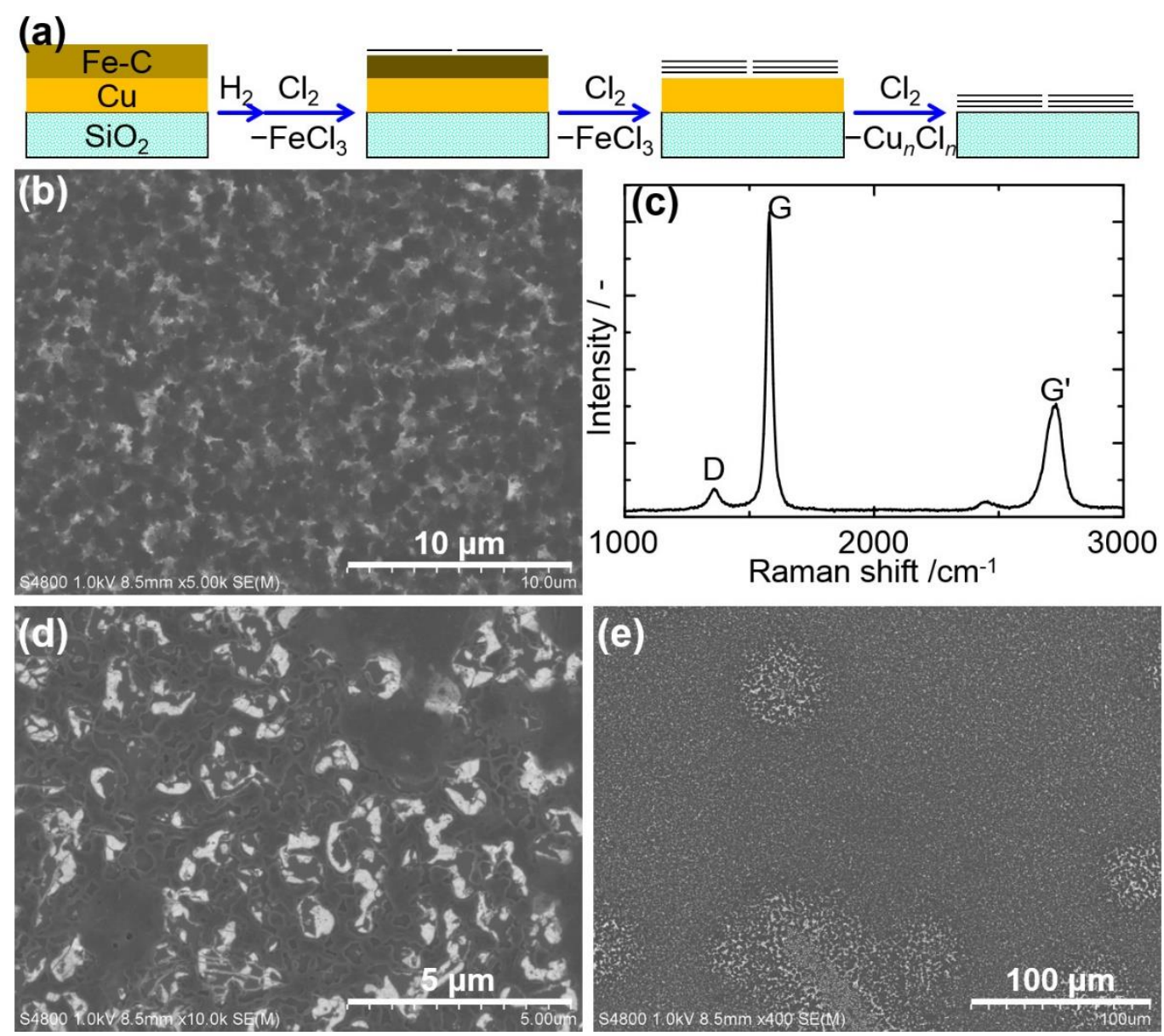

Fig. 6 - (a) Schematic explanation for the etching precipitation of an Fe-C film with a Cu sacrificial underlayer. (b,c) A SEM image and a Raman spectrum of the sample synthesized from a 100 -nm-thick $\mathrm{Fe}-\mathrm{C} / \mathrm{100}$-nm-thick $\mathrm{Cu} / \mathrm{SiO}_{2}$ substrate via etching-precipitation using $0.03 \mathrm{vol} \% \mathrm{Cl}_{2} / \mathrm{Ar}$ for $90 \mathrm{~min}$ at $600{ }^{\circ} \mathrm{C}$. The few-/multi-layer graphene forms uniformly without any voids in some areas. (d) The few-/multilayer graphene with many voids formed in other areas. (e) In this low-magnification image, the few-/multilayer graphene is seen to be non-uniform and the light-colored areas have many voids.

\subsection{Direct fabrication of line patterned graphene/graphite}

In the etching-precipitation method, graphene/graphite can be formed in a micropattern on $\mathrm{SiO}_{2}$ by simply pre-patterning the $\mathrm{Fe}-\mathrm{C}$ mixture film using conventional 
lithographic methods. We investigate such a graphene/graphite formation by pre-patterning the Fe-C films via the lift-off process. First, a ZEP502A resist pattern is fabricated using electron beam lithography on a $\mathrm{SiO}_{2} / \mathrm{Si}$ substrate. Next, the $\mathrm{Fe}-\mathrm{C}$ mixture film is sputter deposited with a thickness of $200 \mathrm{~nm}$. The line-patterned Fe-C film is then fabricated by dissolving the resist and lifting off the $\mathrm{Fe}-\mathrm{C}$ film via ultrasonication in acetone. Etching-precipitation of graphene/graphite is then carried out in $0.05 \mathrm{vol} \% \mathrm{Cl}_{2} / \mathrm{Ar}$ at 5 Torr and $600{ }^{\circ} \mathrm{C}$ for $30 \mathrm{~min}$.

Fig. 7a-c shows the top-view SEM images of the graphene/graphite line patterns directly formed on an $\mathrm{SiO}_{2} / \mathrm{Si}$ substrate. A line pattern with a width greater than $1 \mu \mathrm{m}$ is easily fabricated, showing the applicability of the etching-precipitation method for direct fabrication of electrodes and wirings of graphene/graphite. Some voids are seen in the lines 3 and $1 \mu \mathrm{m}$ in width (Fig. 7a,b) owing to the dewetting process discussed in the previous section, whereas no voids are observed in the narrow lines $0.24 \mu \mathrm{m}$ in width, though some fluctuation in the line width is seen (Fig. 7c). Fig. 7d-g shows the EDS mapping images and the EDS spectrum of the 3- $\mu \mathrm{m}$-wide graphene/graphite line. The line is found to be composed of $\mathrm{C}$ and has no Fe signal, demonstrating the formation of metal-free (C > 99 at\%) graphene/graphite lines. 

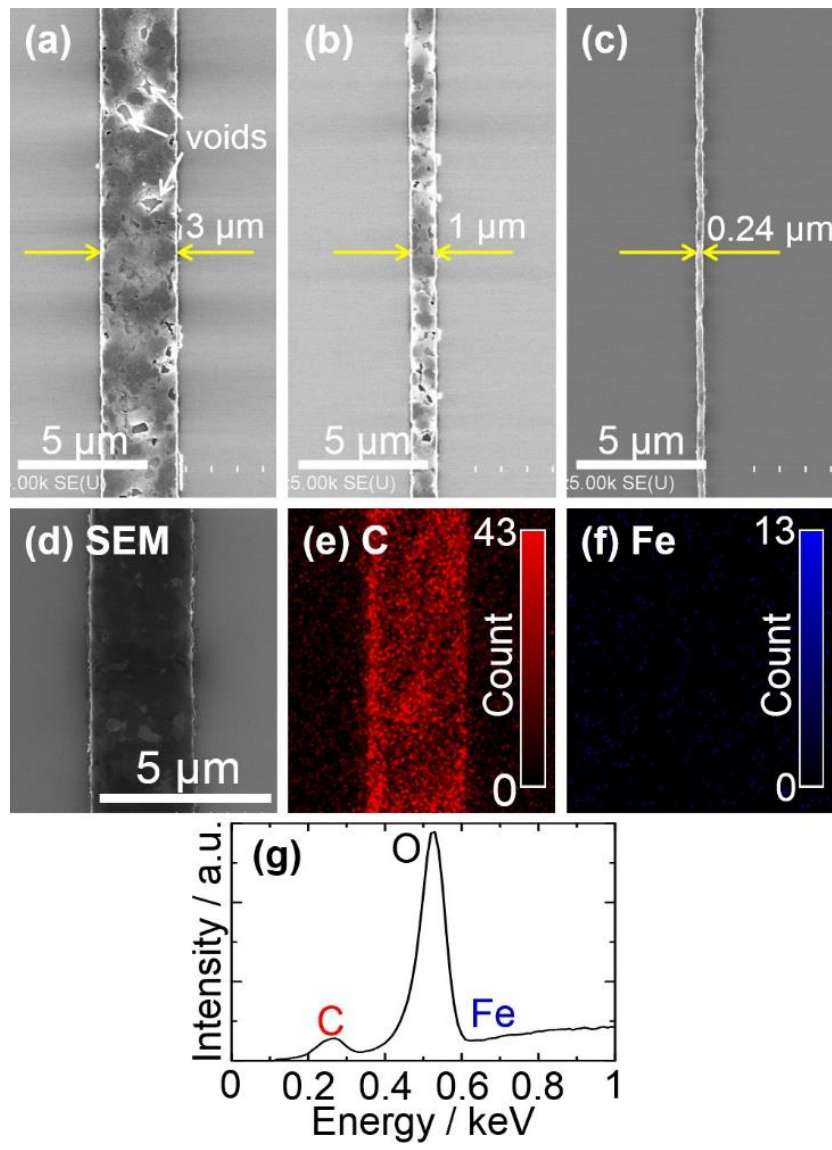

Fig. 7 - SEM images of the graphene/graphite lines directly formed from the Fe-C lines that are $200 \mathrm{~nm}$ in thickness and (a) 3, (b) 1 , and (c) $0.2 \mu \mathrm{m}$ in width, grown on $\mathrm{a} \mathrm{SiO}_{2} / \mathrm{Si}$ substrate. (d) A plan-view SEM image and (e,f) corresponding EDS mapping images,

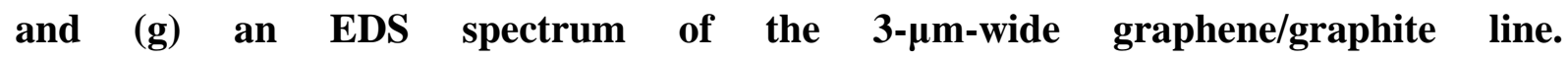
Etching-precipitation was performed using 0.1 vol\% $\mathrm{Cl}_{2}$ at 5 Torr for $90 \mathrm{~min}$. The $\mathrm{O}$ peak in the EDS spectrum (g) originates from the $\mathrm{SiO}_{2}$ underlayer.

Further characterization of these graphene/graphite line patterns is made, and Fig. 8a-c shows the AFM images of the same lines shown in Fig. 7. The expected thickness and layer number of these lines are around $5 \mathrm{~nm}$ and 14 layers, respectively, as estimated from the data of the continuous film sample (Fig. 4a). The 3- $\mu$ m-wide line (Fig. 8a) has a wide plateau that is very thin in the center, but with edges as thick as $200 \mathrm{~nm}$ on both sides that are much greater than the expected thickness of $5 \mathrm{~nm}$. Moreover, the narrower lines (e.g., 1- and 
0.24- $\mu$ m-wide lines in Fig. 8b,c) have an unexpectedly large thickness in the whole region. Fig. $8 \mathrm{~d}-\mathrm{f}$ shows the cross-sectional SEM images of the same graphene/graphite lines. The $3-\mu \mathrm{m}$-wide line has a cross-section that resembles a crumpled arch or a half-dumbbell, whereas the narrower lines ( 1 and $0.3 \mu \mathrm{m}$ in width) have a tubular cross-section. The possible mechanism for these cross-sectional shapes is schematically shown in Fig. 8j. First, the line patterns dewet during annealing to produce a round cross-sectional shape. Then, during the etching of $\mathrm{Fe}$, graphene precipitation takes place on both the top surface and sidewalls of the line patterns possessing the round cross-section. If the contact angle between the $\mathrm{Fe}-\mathrm{C}$ and $\mathrm{SiO}_{2}$ is larger than $90^{\circ}$, the bottom face of the line is also exposed to the vapor phase, resulting in the etching-precipitation over the whole surface of the lines. When the line is narrow, a tubular structure forms, with an expected wall thickness of 7 layers (half of that for uniform films shown in Fig. 4) in this sample. However, as the line width increases, the thin wall of the wide tube is unstable and collapses to yield the crumpled-arch or half-dumbbell shaped cross-sections. Such structures may be attractive when one aims to fill in any materials, but need to be avoided when highly conductive lines are needed because such a structure results in a volume resistivity increase. To obtain ribbon-shaped lines, further study is needed to overcome the dewetting of $\mathrm{Fe}-\mathrm{C}$ lines and graphene precipitation from the sidewalls of the lines. 

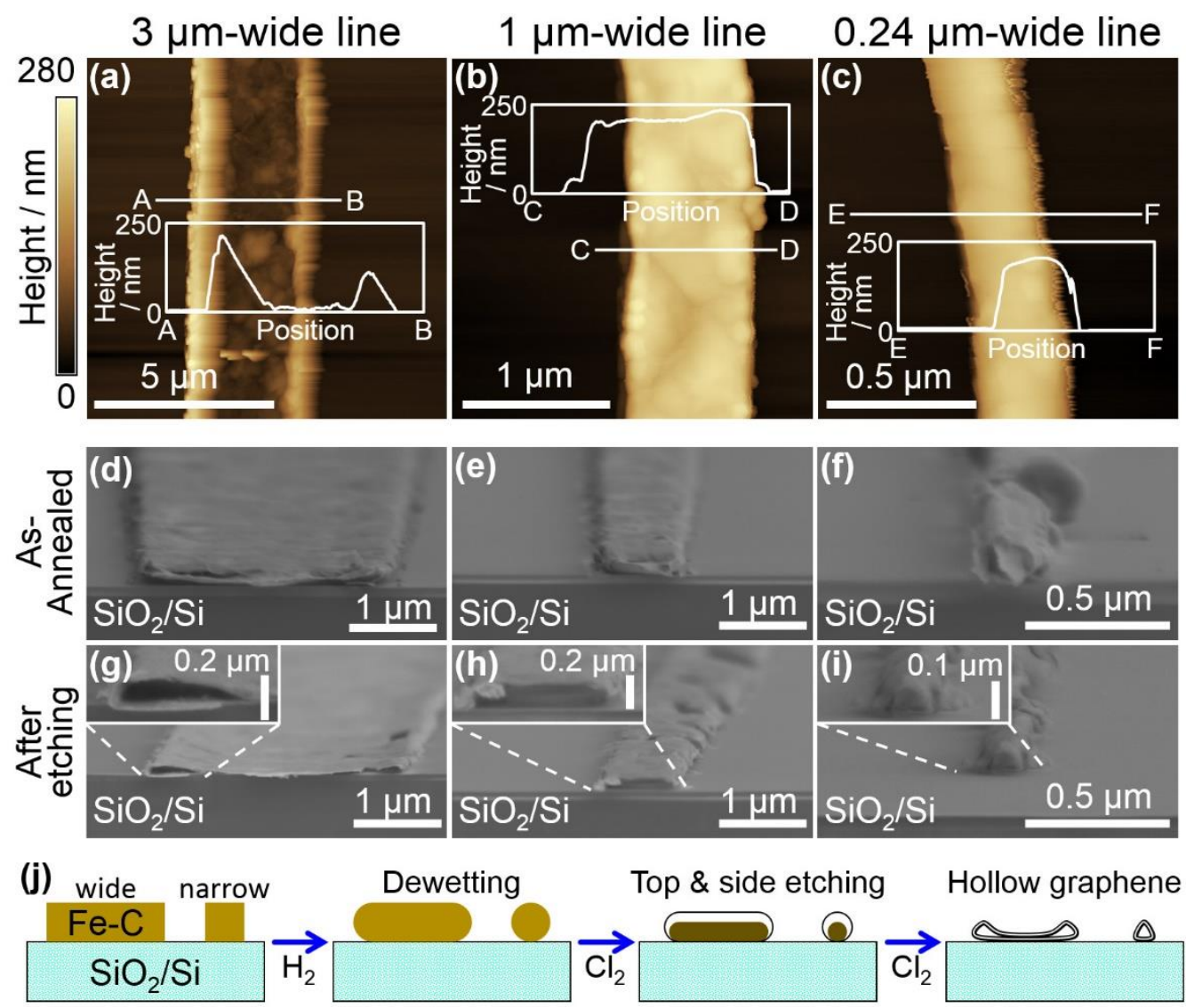

Fig. 8 - Characterization of the graphene/graphite lines shown in Fig. 7. (a-c) AFM images with overlaid height profiles of the graphene lines. Cross-sectional SEM images of (d-f), the as-annealed Fe-C lines, and (g-i) those after etching precipitation (as-synthesized graphene/graphite lines). (j) Schematic of the formation mechanism of the hollow structures in the graphene/graphite lines.

The carrier type, carrier concentration, and carrier mobility of the few-layer graphene synthesized on a quartz glass substrate were determined at $25{ }^{\circ} \mathrm{C}$ using a Hall effect measurement apparatus (TOYO, ResiTest 8400, Tokyo, Japan). As summarized in Table S1, our few-layer graphene film ( $88 \%$ transparency, corresponding to average layer number of 4-5) synthesized on a quartz glass substrate had a p-type carrier with a concentration of $3.2-5.0 \times 10^{20} \mathrm{~cm}^{-3}$ and had a Hall mobility of $82 \mathrm{~cm}^{2} \mathrm{~V}^{-1} \mathrm{~s}^{-1}$ at $25^{\circ} \mathrm{C}$. The resistivity needs be reduced further to be used as wirings in LISs. Improving the film quality as well as doping 
are important, which is now under investigation.

\section{Conclusions}

In summary, we propose an "etching-precipitation" method that realizes the fabrication of metal-free few-/multi-layer graphene directly on dielectric substrates. The 6-7-layer graphene directly fabricated on a quartz glass substrate at $650{ }^{\circ} \mathrm{C}$ exhibits an $86 \%$ optical transmittance and a 340-460 $\Omega /$ sq. sheet resistance, corresponding to an $80-140 \mu \Omega$ $\mathrm{cm}$ volume resistivity. The number of layers is linearly controlled using only the initial feed of carbon, which is accomplished by changing the thickness of the initial Fe-C mixture films. Sub- $\mu \mathrm{m}$-wide line patterns are also fabricated directly onto a $\mathrm{SiO}_{2} / \mathrm{Si}$ substrate by pre-patterning the $\mathrm{Fe}-\mathrm{C}$ mixture using a conventional lithographic method, although the graphene/graphite lines possess a hollow structure. There is a trade-off relationship between the domain growth and the formation of voids, with an enhanced domain size and void formation at elevated temperatures. Furthermore, another trade-off relationship exists between the graphene layer number and the formation of voids, with a reduced number of layers and reduced graphene surface coverage for thinner initial $\mathrm{Fe}-\mathrm{C}$ films. Therefore, to produce void-free graphene with larger domain sizes and a reduced number of layers, we must avoid the dewetting of the Fe-C film prior to the precipitation of graphene. Further studies are ongoing towards such improvements by seeking a suitable underlayer such as $\mathrm{Cu}$ for the $\mathrm{Fe}-\mathrm{C}$ film.

For graphene applications using both conducting and semi-conducting device elements, a transfer-free process yielding graphene directly on dielectric substrates is in high demand. Our etching-precipitation method is compatible with substrates of any size, and would be promising for few-/multi-layer graphene fabrication at both the macro- and micro-scales. 


\section{Acknowledgements}

The authors thank T. Takeuchi and I. Yuito at Waseda University for fabrication of resist patterns by electron-beam lithography, which was performed on Nanotechnology Platform Program supported by MEXT, Japan. The authors thank Mr. H. Asakura at TOYO Corporation for the Hall effect measurement and Ms. Y. Akiba at Waseda University for some AFM measurements. This work was financially supported by PRESTO (no. 3130) from JST, Japan and a Grant-in-Aid for Scientific Research on Innovative Areas (no. 25107002) from MEXT, Japan. 


\section{REFERENCES}

[1] Bianco A, Cheng HM, Enoki T, Gogotsi Y, Hurt RH, Koratkar N, et al. All in the graphene family - a recommended nomenclature for two-dimensional carbon materials. Carbon 2013;65:1-6.

[2] Novoselov KS, Geim AK, Morozov SV, Jiang D, Zhang Y, Dubonos SV, et al. Electric field effect in atomically thin carbon films. Science 2004:306(5696);666-9.

[3] Castro EV, Novoselov KS, Morozov SV, Peres NMR, Lopes dos Santos JMB, Nilsson J, et al., Biased bilayer graphene: semiconductor with a gap tunable by the electric field effect. Phys Rev Lett 2007:99(21);216802-1-4.

[4] Zhang Y, Tang T, Girit C, Hao Z, Martin MC, Zettl A, et al. Direct observation of a widely tunable bandgap in bilayer graphene. Nature 2009;459(7248):820-3.

[5] Chen J, Jang C, Xiao S, Ishigami M, Fuhrer MS. Intrinsic and extrinsic performance limits of graphene devices on $\mathrm{SiO}_{2}$. Nat Nanotechnol 2008;3(4):206-9.

[6] Li X, Cai W, An J, Kim S, Nah J, Yang D, et al. Large-area synthesis of high-quality and uniform graphene films on copper foils. Science 2009;324(5932):1312-4.

[7] Bae S, Kim H, Lee Y, Xu X, Park J, Zheng Y, et al. Roll-to-roll production of 30-inch graphene films for transparent electrodes. Nat Nanotechnol 2010;5(8):574-8.

[8] Li X, Zhu Y, Cai W, Borysiak M, Han B, Chen D, et al. Transfer of large-area graphene films for high-performance transparent conductive electrodes. Nano Lett 2009;9(12):4359-63.

[9] Vlassiouk I, Regmi M, Fulvio P, Dai S, Datskos P, Eres G, et al. Role of hydrogen in chemical vapor deposition growth of large single-crystal graphene. ACS Nano 2011;5(7):6069-76.

[10] Jacobberger RM, Arnold MS. Graphene growth dynamics on epitaxial copper thin films. Chem Mater 2013;25(6):871-7. 
[11] Li X, Cai W, Colombo L, Ruoff RS. Evolution of graphene growth on Ni and Cu by carbon isotope labeling. Nano Lett 2009;9(12):4268-72.

[12] Ago H, Ogawa Y, Tsuji M, Mizuno S, Hibino H. Catalytic growth of graphene: toward large-area single-crystalline graphene. J Phys Chem Lett 2012;3(16):2228-36.

[13] Yu Q, Lian J, Siriponglert S, Li H, Chen YP, Pei SS. Graphene segregated on Ni surfaces and transferred to insulators. Appl Phys Lett 2008;93(11):113103-1-3.

[14] Kim KS, Zhao Y, Jang H, Lee SY, Kim JM, Kim KS, et al. Large-scale pattern growth of graphene films for stretchable transparent electrodes. Nature 2009;457(7230):706-10.

[15] Reina A, Jia X, Ho J, Nezich D, Son H, Bulovic V, et al. Large area, few-layer graphene films on arbitrary substrates by chemical vapor deposition. Nano Lett 2009;9(1):30-5.

[16] Xue Y, Wu B, Guo Y, Huang L, Jiang L, Chen J. Synthesis of large-area, few-layer graphene on iron foil by chemical vapor deposition. Nano Res 2011;4(12)1208-14.

[17] An H, Lee W, Jung J. Graphene synthesis on Fe foil using thermal CVD. Curr Appl Phys 2011;11(4):S81-5.

[18] Huang L, Chang QH, Guo GL, Liu Y, Xie YQ, Wang T, et al. Synthesis of high-quality graphene films on nickel foils by rapid thermal chemical vapor deposition. Carbon 2012;50(2):551-6.

[19] Kondo D, Sato S, Yagi K, Harada N, Sato M, Nihei M, et al. Low-temperature synthesis of graphene and fabrication of top-gated field effect transistors without using transfer processes. Appl Phys Express 2010;3(2):025102-1-3.

[20] Kondo D, Yagi K, Sato M, Nihei M, Awano Y, Sato S, et al. Selective synthesis of carbon nanotubes and multi-layer graphene by controlling catalyst thickness. Chem Phys Lett 2011;514(4-6):294-300.

[21] Wang Y, Zheng Y, Xu X, Dubuisson E, Bao Q, Lu J, et al. Electrochemical delamination of CVD-growth graphene film: toward the recyclable use of copper catalyst. ACS Nano 
2012;5(12):9927-33.

[22] Gao L, Ren W, Xu H, Jin L, Wang Z, Ma T, et al. Repeated growth and bubbling transfer of graphene with millimetre-size single-crystal grains using platinum. Nat Commun 2012;3:699-1-7.

[23] Peng Z, Yan Z, Sun A, Tour JM. Direct growth of bilayer graphene on $\mathrm{SiO}_{2}$ substrates by carbon diffusion through nickel. ACS Nano 2011;5(10):8241-7.

[24] Yang Z, Peng Z, Sun Z, Yao J, Zhu Y, Liu Z, et al. Growth of bilayer graphene on insulating substrates. ACS Nano 2011;5(10):8187-92.

[25] Shin H, Choi WM, Yoon S, Han GH, Woo YS, Kim ES, et al. Transfer-free growth of few-layer graphene by self-assembled monolayers. Adv Mater 2011;23(38):4392-7.

[26] Kumar S, McEvoy N, Kim H, Lee K, Peltekis N, Rezvani E, et al. CVD growth and processing of graphene for electronic applications. Phys. Status Solidi B $2011 ; 248(11): 2604-8$.

[27] Su C, Lu A, Wu C, Li Y, Liu K, Zhang W, et al. Direct formation of wafer scale graphene thin layers on insulating substrates by chemical vapor deposition. Nano Lett 2011;11(9):3612-16.

[28] Sun J, Lindvall N, Cole MT, Teo KBK, Yurgens A. Large-area uniform graphene-like thin films grown by chemical vapor deposition directly on silicon nitride. Appl Phys Lett 2011;98(25):252107-1-3.

[29] Xu SC, Man BY, Jiang SZ, Chen CS, Yang C, Liu M, et al. Direct synthesis of graphene on $\mathrm{SiO} 2$ substrates by chemical vapor deposition. CrystEngComm 2013;15(10):1840-4.

[30] Takahashi K, Yamada K, Kato H, Hibino H, Homma Y. In situ scanning electron microscopy of graphene growth on polycrystalline Ni substrate. Surf Sci 2012;606(78):728-32.

[31] Weatherup RS, Bayer BC, Blume R, Ducati C, Baehtz C, Schlogl R, Hofmann S. In situ 
characterization of alloy catalysts for low-temperature graphene growth. Nano Lett. 2011;11(10):4154-60.

[32] Weatherup RS, Dlubak B, Hofmann S. Kinetic control of catalytic CVD for high-quality graphene at low temperatures. ACS Nano 2012;6(11):9996-10003.

[33] Wert CA. Diffusion coefficient of C in $\alpha$-iron. Phys Rev 1950;79(4):601-5. 


\section{Figure captions}

Fig. 1 - Schematic comparing the CVD and "etching-precipitation" processes. (a) In a CVD process using a carbon-insoluble metal catalyst such as $\mathrm{Cu}$, the graphene growth proceeds through the surface reaction [11]. (b) In a CVD process using a carbon-soluble metal catalyst such as $\mathrm{Ni}$, the graphene growth proceeds through carbon supersaturation and precipitation from the Ni-C solid solution upon cooling [11], as well as through the surface reaction [30-32]. (c) In the etching-precipitation of the metal-C mixed film (e.g., metal=Fe), the graphene growth proceeds through carbon supersaturation and precipitation by removing solid-solvent Fe, resulting in Fe-free graphene directly on the substrates.

Fig. 2 - The (a) photograph, (b) Raman spectrum, (c) SEM image, and (d) AFM image of a typical multi-layer graphene sample synthesized by the etching-precipitation method. The 100-nm-thick Fe-C mixture film on a quartz glass substrate is etched under 0.05 vol\% $\mathrm{Cl}_{2}$ at 5 Torr and $650{ }^{\circ} \mathrm{C}$ for $60 \mathrm{~min}$. The graphene is $86 \%$ transparent, corresponding to six to seven layers, $3.4 \mathrm{~nm}$ in thickness, and a sheet resistance of 340 $460 \Omega / \mathrm{sq}$., which corresponds to $80-140 \mu \Omega \mathrm{cm}$ volume resistivity (see the main text). The Raman spectrum is taken at $488 \mathrm{~nm}$ for excitation. The AFM image is obtained from graphene transferred onto a $\mathrm{SiO}_{2} / \mathrm{Si}$ substrate with a flat surface.

Fig. 3 - Few-/multi-layer graphene synthesized on $\mathrm{SiO}_{2} / \mathrm{Si}$ from a 100 -nm-thick Fe-C film by etching-precipitation using $0.05 \mathrm{vol} \% \mathrm{Cl}_{2}$ at 5 Torr for $15 \mathrm{~min}$ at various temperatures. (a-g) Plan-view SEM images. (h) Raman spectra using an excitation wavelength of 488 nm. (i) The surface coverage and the $I_{\mathrm{G}} / I_{\mathrm{D}}$ ratios of the graphene films synthesized at 400-700 ${ }^{\circ} \mathrm{C}$.

Fig. 4 - (a) Optically scanned images of few-/multi-layer graphene grown from 20 to 
200-nm-thick Fe-C at $600{ }^{\circ} \mathrm{C}$ using 0.05 vol\% $\mathrm{Cl}_{2}$ at 5 Torr on quartz glass substrates of $15 \times 15 \mathrm{~mm}^{2}$. (b) Optical transmittance (blue open squares) and layer number (red open circles) of the graphene on quartz glass substrates shown in (a). The blue straight line shows the estimated layer number when considering the mass balance in $\mathrm{C}$ for the reduction of iron oxide and graphene formation (see the main text). (c) Plan-view SEM images of the few-/multi-layer graphene films grown from 20- to 200-nm-thick $\mathrm{Fe}-\mathrm{C}$ at $600{ }^{\circ} \mathrm{C}$ on $\mathrm{SiO}_{2} / \mathrm{Si}$ substrates. (d) Surface coverage of the graphene films on $\mathrm{SiO}_{2} / \mathrm{Si}$ substrates shown in (c).

Fig. 5 - (a) SEM images of the 100-nm-thick Fe-C film partially etched by $0.05 \mathrm{vol} \% \mathrm{Cl}_{2}$ for a short time of 2 min at $600{ }^{\circ} \mathrm{C}$, and EDS mapping images of (b) $\mathrm{C}$ and (c) $\mathrm{Fe}$ on the surface. Island-like structures of the Fe-C mixture, voids (surrounded by yellow broken curves), and graphene surrounding the voids (the regions between yellow and blue broken curves) are observed. (d) A schematic for the formation mechanism of few-/multi-layer graphene and voids.

Fig. 6 - (a) Schematic explanation for the etching precipitation of an $\mathrm{Fe}-\mathrm{C}$ film with a $\mathrm{Cu}$ sacrificial underlayer. (b,c) A SEM image and a Raman spectrum of the sample synthesized from a 100-nm-thick Fe-C/ 100-nm-thick $\mathrm{Cu} / \mathrm{SiO}_{2}$ substrate via etching-precipitation using $0.03 \mathrm{vol} \% \mathrm{Cl}_{2} / \mathrm{Ar}$ for $90 \mathrm{~min}$ at $600{ }^{\circ} \mathrm{C}$. The few-/multi-layer graphene forms uniformly without any voids in some areas. (d) The few-/multilayer graphene with many voids formed in other areas. (e) In this low-magnification image, the few-/multilayer graphene is seen to be non-uniform and the light-colored areas have many voids.

Fig. 7 - SEM images of the graphene/graphite lines directly formed from the Fe-C lines that are $200 \mathrm{~nm}$ in thickness and (a) 3, (b) 1 , and (c) $0.2 \mu \mathrm{m}$ in width, grown on $\mathrm{a} \mathrm{SiO}_{2} / \mathrm{Si}$ 
substrate. (d) A plan-view SEM image and (e,f) corresponding EDS mapping images, and (g) an EDS spectrum of the 3- $\mu$ m-wide graphene/graphite line. 0.05 vol\% $\mathrm{Cl}_{2}$ at 5 Torr is used for etching precipitation. The O peak in the EDS spectrum (g) originates from the $\mathrm{SiO}_{2}$ underlayer.

Fig. 8 - Characterization of the graphene/graphite lines shown in Fig. 7. (a-c) AFM images with overlaid height profiles of the graphene lines. Cross-sectional SEM images of $(\mathrm{d}-\mathrm{f})$, the as-annealed $\mathrm{Fe}-\mathrm{C}$ lines, and $(\mathrm{g}-\mathrm{i})$ those after etching precipitation (as-synthesized graphene/graphite lines). (j) Schematic of the formation mechanism of the hollow structures in the graphene/graphite lines. 